\title{
Balkanologie
}

Balkanologie Revue d'études pluridisciplinaires

Vol. IX, n $1-2 \mid 2005$

Volume IX Numéro 1-2

\section{Le meurtre du prêtre}

Acte fondateur de la mobilisation nationaliste albanaise à l'aube de la révolution Jeune Turque

The murder of the priest. Founding act of the Albanian nationalist mobilisation on the eve of the Young Turks revolution

\section{Nathalie Clayer}

\section{CpenEdition}

\section{Journals}

Édition électronique

URL : http://journals.openedition.org/balkanologie/575

DOI : $10.4000 /$ balkanologie. 575

ISSN : 1965-0582

Éditeur

Association française d'études sur les Balkans (Afebalk)

Édition imprimée

Date de publication : 1 décembre 2005

ISSN : 1279-7952

\section{Référence électronique}

Nathalie Clayer, «Le meurtre du prêtre », Balkanologie [En ligne], Vol. IX, n 1-2 | 2005, mis en ligne le 13 janvier 2010, consulté le 17 décembre 2020. URL : http://journals.openedition.org/balkanologie/575 ; DOI : https://doi.org/10.4000/balkanologie.575 


\title{
LE MEURTRE DU PRÊTRE ACTE FONDATEUR DE LA MOBILISATION NATIONALISTE ALBANAISE À L'AUBE DE LA RÉVOLUTION JEUNE TUROUE
}

\author{
Nathalie Clayer*
}

En septembre 1906, une çeta (bande) albanaise assassine le métropolite orthodoxe de Korçë, ville située à l'ouest de Bitola, dans une région fortement albanophone. À première vue, on pourrait voir dans ce meurtre du chef local de la communauté orthodoxe perpétré par un groupe formé en majeure partie de musulmans la manifestation tangible d'un affrontement religieux. En réalité, cet acte de violence, replacé dans un cadre plus général, doit être interprété sur un autre plan, celui du nationalisme albanais naissant.

Pour le comprendre, je reviendrai d'abord sur le contexte général qui prévalait dans cette région dans les premières années $d u X X^{\text {ème }}$ siècle, à savoir celui de l'affrontement de plus en plus âpre, et de plus en plus violent, entre les différentes propagandes - grecque, bulgaro-macédoniennes, aroumaine et serbe-, et celui du renforcement d'une composante mishellène dans les constructions identitaires albanaises d'alors. Puis, j'analyserai l'émergence de la guérilla albanaise et les raisons du meurtre du prētre. Enfin, je montrerai comment les Albanais chrétiens orthodoxes utilisèrent eux-aussi cet événement, mais plus d'un an après les faits, lorsqu'ils décidèrent de s'émanciper du Patriarcat de Constantinople.

\section{ÊTRE ALBANAIS, OU S'OPPOSER À L'HELLÉNISME EN ÉPIRE}

Dans les premières années du XXème siècle, la Macédoine devient le théâtre d'une compétition acharnée entre différentes propagandes nationales. Des comités secrets, des bandes de guérilleros s'y affrontent depuis plusieurs an- 
nées. La lutte se manifeste sur divers plans qui s'entremèlent : l'éducation (des réseaux scolaires concurrents sont mis en place) ; l'économie (des réseaux commerciaux nationaux sont créés, des boycotts sont lancés) ${ }^{1}$; l'Église (différents réseaux ecclésiastiques se battent pour attirer les fidèles : le réseau du Patriarcat, soutien de l'hellénisme, le réseau exarchiste bulgare, etc.) et la guérilla, avec le développement de bandes regroupant des jeunes gens prêts à faire usage de la force ${ }^{2}$.

Dans les confins sud-ouest de la Macédoine, c'est-à-dire à la frontière entre la Macédoine et l'Épire, la propagande la plus puissante est alors certainement la propagande grecque. Au sein des milieux patriarchistes, l'hellénisme tend d'ailleurs de plus en plus à faire coïncider orthodoxie et grécité. La " grécisation" de la hiérarchie religieuse dépendant du Patriarcat et son raidissement vis-à-vis de l'usage de toute autre langue que le grec (slave, albanais ou aroumain) se font de plus en plus nets. Les anathèmes, les menaces d'excommunications deviennent de plus en plus courantes à l'encontre de ceux qui font la promotion de ces autres idiomes. En 1905, Mihal Grameno, un jeune intellectuel de Korçë vivant en Roumanie, dénonce dans une " pièce nationale " la "malédiction de la langue albanaise " par les prêtres, "espions de la Grèce "3.

En allant vers l'est et le nord, l'hellénisme se heurte aux propagandes bulgaro-macédonienne et, dans une moindre mesure, serbe. Mais il voit aussi s'affirmer une autre opposition : le roumano-aroumanisme4. Les Aroumains, qui disposaient déjà d'un réseau scolaire, commencent en 1904 à réclamer leur autonomie vis-à-vis du millet-i rum. En 1905, ils parviennent à obtenir un irade imperial leur octroyant un statut particulier, qui leur permet de former un " quasi-millet ", à cela près que le Patriarcat renâcle à reconnaître l'autonomie religieuse à laquelle ils aspirent 5 .

Dans ce contexte, il est logique que certains albanistes songent également à une autonomie, voire à une émancipation de la tutelle du Patriarcat. Une

\footnotetext{
${ }^{1}$ A ce sujet, voir Gounaris (Basil C.), "From Peasants into Urbanites, from Village into Nation : Ottoman Monastir in the Early Twentieth Century ", European History Quarterly, 31 (1), 2001, pp. 55-58.

2 Adanir (Fikret), Die makedonische Frage. Ihre Entstehung und Entwicklung bis 1908 (La question macédonienne. Sa formation et son développement jusqu'en 1908), Wiesbaden : Franz Steiner Verlag, 1979 [Frankfurter Historische Abhandlungen, Band 2o].

3 Mallkimi i gjuhes shqipe, prej M.G., Pjese kombiare me vjershe me tre pamje, ngjare ne Korçe me 1886 (La malédiction de la langue albanaise, par M.G., Pièce nationale en vers et en trois actes, se passant à Korçë en 1886), Bukuresht : Shyt. N.N. Voicu, 1905

4 Je reprends ici l'expression de M. D. Peyfuss (Peyfuss (Max Demeter), Die aromunische Frage. Ihre Entwicklung van der Ursprüngen bis zum Frieden von Bukarest (1913) und die Haltung Österreich-Ungarns (La question aroumaine. Son développement depuis les origines jusqu'à la paix de Bucarest (1913) et l'attitude de l'Autriche-Hongrie), Wien : Böhlau, 1974).
}

5 Peyfuss (Max Demeter), op.cit., p. 85 ss. et pp. 112-113. 
telle attitude n'est cependant encore que très marginale. La plupart des albanophones orthodoxes, voire même certains musulmans albanophones, adhèrent à l'hellénisme. Si une albanité au sens moderne se construit peu à peu chez eux, c'est en restant généralement dans le giron de l'hellénisme ${ }^{6}$. Dans certains milieux orthodoxes influencés par les évolutions qui se manifestent chez les Aroumains, on commence cependant à vouloir promouvoir l'usage de la langue albanaise dans le domaine religieux.

Et c'est, au reste, dans un environnement mi-albanais mi-aroumain qu'émerge la figure d'un premier pope ouvertement albaniste. Kristo Negovani, ainsi se nomme-t-il, est né en 1875, à Negovan, un village mixte albanophone et aroumanophone de Macédoine occidentale, situé près de Florina. Fils d'un commerçant faisant affaires à Athènes, il étudie plusieurs années dans un lycée de la capitale grecque. En 1891, à la suite de l'assassinat de son père par des bandits, il doit commencer à travailler comme instituteur dans le réseau des écoles grecques de la région. Puis, en 1894, il émigre en Roumanie, à Brăila, où se trouvent en émigration de nombreux hommes de Negovan, et exerce le métier de charpentier. Dans la diaspora, il est sensibilisé à l'albanisme qui se développe alors en étroit lien avec le roumano-aroumanisme. Trois ans plus tard, au milieu de la crise des annees 1890 donc, il rentre dans son village natal. Le jeune homme, qui ne s'est pourtant pas destiné jusque là à la carrière religieuse, se fait alors rapidement ordonner prêtre et commence à exercer son ministère, faisant des messes en albanais et enseignant aux enfants leur langue maternelle. Surtout, à partir de 1899, il se met à publier des ouvrages en albanais. Il s'agit de fables et de poèmes didactiques, de textes d'instruction religieuse, ainsi que d'articles parus dans l'almanach Kalendari Kombiar et dans le journal Drita (tous deux édités à Sofia), souvent sous la forme de prêches. Il y prend position contre la propagande grecque et s'élève même contre les mariages avec des "éléments étrangers ". Au début de l'année 1905, avec son frère lui aussi pope et trois autres villageois, il est victime d'une bande grecque et devient le premier " martyr " de la cause nationale albanaise?

De fait, dans les mois qui suivent, ce meurtre ne manque pas d'être dénoncé par les albanistes dans leurs périodiques et d'être attribué, au moins en partie, à l'Église patriarchiste. Dans les pages de Drita (La lumière), le journal albaniste le plus important de l'époque ${ }^{8}$, le clergé orthodoxe est vigoureusement attaqué. Il est taxé de barbare. Il est rendu responsable, au même titre

${ }^{6}$ A ce sujet, voir mon ouvrage Aux origines du nationalisme albanais à paraitre chez Karthala en 2006.

7 Cf. Fullani (Dhimitër). "Papa Kristo Negovani ", Buletin i Universitetit Shtetëror të Tiranës, Seria Shkencat Shoqërore, 2, 1960, pp. 188-221; et Elsie (Robert), History of Albanian Literature, 2 vol., New York Columbia Univ. Press, 1995. pp. 284-286.

${ }^{8}$ Ce journal était édité à Sofia par Shahin Kolonja. Il parut entre 1901 et 1908 (cf. Sokolova (Bojka). Albanski väzrożdenski pećat $v$ Bälgarija (La presse de la renaissance albanaise en Bulgarie), Sofia Bảlgarska Akademija na Naukite, 1979). 
que la Grèce, de l'assassinat de Papa Kristo Negovani. Les métropolites sont présentés comme les instigateurs des assassinats. En particulier, le despote de Korçë, Photios, est fustigé, parce qu'il a ordonné aux commerçants de la ville un boycott contre les Aroumains de Plasë (un village des environs de Korçë) opposés à l'hellénisme, parce qu'il a refusé d'enterrer certains albanistes ou encore parce qu'il n'a pas voulu célébrer un mariage dans la famille Kosturi, connue pour sa position en faveur de l'albanisme.

De façon plus générale, en feuilletant le journal Drita, on s'aperçoit à quel point la rigidité de la haute hiérarchie orthodoxe, l'association de plus en plus stricte de la langue grecque à l'orthodoxie, le développement des bandes grecques contre les " mouvements " bulgare et aroumain, ainsi que les velléités aroumaines ont, à cette époque, pesé sur le développement du nationalisme albanais aux confins de la Macédoine et de l'Épire. Dans les colonnes de ce périodique, les anathèmes lancés par les popes contre les albanistes sont dénoncés. Les interdictions faites aux orthodoxes d'aller dans des écoles autres que grecques, y compris des écoles françaises, sont critiquées. Face aux affirmations des popes présentant la langue albanaise comme une langue " maudite par Dieu ", par opposition au grec " langue des Dieux ", les auteurs de certains articles rétorquent que le Christ n'était pas grec. Ils mettent en avant l'esprit de la Pentecôte et la capacité donnée aux Apôtres de diffuser les Évangiles en utilisant les différentes langues des peuples, un argument qui sera souvent utilisé pour justifier l'emploi de l'albanais à l'église? 9 .

D'autre part, c'est en rapport étroit avec la Roumanie et les Aroumains que l'idée d'une autonomie religieuse fait son apparition au détour de certains articles. En mai 1903, Kristaq/Kristo Dako évoque déjà l'idée d'une Église pour les Albanais en Roumanie ${ }^{10}$. Dès le printemps 1904, il est question des efforts des Aroumains de se détacher de l'Église grecque. En 1905, on salue la récompense de leurs efforts et leur séparation d'avec les Grecs. L'année suivante, un auteur explique qu'il ne faut pas abandonner l'orthodoxie, mais les églises et les écoles "étrangères ", c'est-à-dire "non-albanaises " et qu'il faut traduire les livres d'église en albanais. À l'automne 1906, il est annoncé dans le journal que, grâce à l'action aroumaine, une église particulière a été ouverte à Korçë spécialement pour les "Albano-Valaques "11.

9 Voir également Luarasi (Petro N.), Mallkim i Shkronjavet Shqipe dhe çperfolja e Shqipetarit (La malédiction des lettres albanaises et le dénigrement de l'Albanais), Manastir: Shtyp. Tregëtare nëkombëtare, 1911.

10 Les diasporas albanaises anciennes (Grèce, Italie), ainsi que celles qui se constituèrent au cours du $\mathrm{XIX}^{\text {eme }}$ siècle, comme en Roumanie, en Bulgarie ou en Égypte, ou même au début du XXème siècle en Amérique, furent des lieux particulièrement propices au développement de l'albanisme et, en particulier, aux activitès d'édition (à ce sujet, voir mon ouvrage à paraitre, cité en note 6).

${ }^{11}$ Sur tous ces sujets, voir en particulier les numéros 7 (de 1902), 26 (de 1903), 41 (de 1904), 58, 59, 60, 62 et 71 (de 1905), 79.82 et 83 (de 1906) de Drita. 
Mais les chrétiens orthodoxes ne sont pas les seuls à réagir. À la même période, dans le contexte général de lutte entre comités secrets et bandes, des Albanais musulmans décident eux aussi de créer leurs propres organisations.

\section{LA FORMATION DE LA PREMIÈRE ÇETA ALBANAISE}

La mobilisation albaniste dans l'Empire et la création d'un comité secret albanais en 1905, à l'image de ce que d'autres avaient fait, est essentiellement l'œuvre de musulmans. Les cinq personnes qui décident de passer à l'action et constituent la cellule centrale du comité à Bitola, pendant une nuit de ramadan du mois de novembre 1905 , sont en effet les suivantes. Il y a deux musulmans appartenant à l'ancienne génération : Halid bey Bërzeshta, colonel et chef du corps des pharmaciens de la III ${ }^{\mathrm{e}}$ armée, et Fehim bey Zavalani, un propriétaire terrien. Il y a ensuite deux musulmans de la jeune génération : Bajram Fehmi (ou Bajo) Topulli, vice-directeur de l'idadiye, et Sejfeddin (Sejfi) Vllamasi, vétérinaire de la municipalité. Il y a enfin un chrétien, mais un chrétien protestant : le fameux missionnaire Gjergj Oiriazi, par ailleurs drogman du consulat austro-hongrois. Ce dernier se serait d'ailleurs retiré par la suite, et aurait été remplacé par un musulman, Jashar Bitincka, un collègue de Bajo Topulli, professeur à l'idadiye.

D'après un rapport consulaire austro-hongrois, ce sont également généralement des musulmans, notables ou fonctionnaires, qui ont formé des cellules du comité dans les villes voisines. À Starovë, il y aurait Husrev bey, le notable le plus influent de la région, Mustafa efendi, fonctionnaire de la dette ottomane et Jashar bey ; à Ohrid, Mehdi bey Frashëri qui occupe le poste de kaymakam ; et à Prilep, son collègue Shevket bey Frashëri, lui aussi kaymakam. D'après Sejfi Vllamasi, plusieurs kavas travaillant pour des consulats à Skopje, Bitola et Salonique - tous originaires de Kolonjë comme lui - comptent également parmi les membres du comité. À Bitola, il y a le lieutenant Nafiz Ohri et Idriz Gjakova, à l'époque fournisseur de nourriture pour l'armée. Sulejman Starova, fonctionnaire des finances à Naslić/Anaselitsa (Neapolis), en faisait aussi partie, de même que Sali Butka de Kolonjë. Sejfi Vllamasi ne mentionne qu'un seul orthodoxe : Nuçi Naçi qui avait autrefois enseigné l'albanais à l'école albanaise de Korçë avant d'être arrēté par les autorités ottomanes ${ }^{12}$.

\footnotetext{
${ }^{12}$ Pepo (Petraq), éd., Kujtime nga lëvizja për çlirimin kombetar (1878-1912) (Souvenirs du mouvement pour la libération nationale), Tiranë, 1962, p. 231 ss. ; Vlamasi (Sejfi), Ballafaqime politike në Shqipëri. $1897-1942$ (Kujtime dhe vlerësime historike) (Confrontations politiques en Albanie. 1897-1942 [Souvenirs et appréciations historiques]), Tiranë : Marin Barleti, 1995, pp. 26-28 ; Haus-, Hof- und Staats Archiv (Vienne), PA XIV/14, liasse XI, Prochaska, Monastir, 21/4/1906.
} 
Pour ces Albanais - donc en très large majorité musulmans - dans le contexte macédonien de ce début de $\mathrm{XX}$ ème siècle, entrer dans la compétition politique passe désormais non seulement par la diffusion de journaux et d'imprimés, mais aussi par la formation de comités secrets et la mise sur pied de bandes (çeta). Lorsque Sejfeddin Vllamasi, lui-même membre du comité, évoque le contexte de la création de cette cellule dans ses mémoires, il explique que les rivalités entre Grecs et Bulgares avaient alors atteint un point culminant, ensanglantant la région. Cette anarchie poussait les Grandes Puissances à faire pression sur le sultan pour lui faire accepter la formation d'une commission de contrôle et de nouvelles réformes en Macédoine ${ }^{13}$. D'autre part, des bataillons de chasseurs avaient été mis sur pied afin de lutter contre les bandes qui s'affrontaient dans le but de se partager ultérieurement les "territoires albanais " (c'est ainsi que Sejfi Vllamasi voyait en tout ou en partie la Macédoine). Ces bandes étaient bien organisées. Pour lui, elles étaient soutenues financièrement par les États balkaniques, à leur tour aidés par les Grandes Puissances ${ }^{14}$.

Mais quels sont les buts du nouveau comité ? D'après les statuts qui ont été publiés à Sofia sous deux formes - dans chacun des dialectes geg et tosk ${ }^{15}$-, l'objectif est " la régénération de l'Albanie ". Pour cela, ses membres se doivent de semer la fraternité, l'amour et l'union, de répandre la voie de la civilisation par le moyen de livres, d'envoyer des émissaires aux quatre coins de l'Albanie afin de répandre de telles idées, et d'enrôler des gens dans les montagnes pour aider le comité à œuvrer par tous les moyens pour le progrès de la nation, " afin d'échapper au joug et aux ténèbres " dans lesquels les Albanais se trouvent. Il s'agit aussi "d'œuvrer contre le danger qui cerne l'Albanie ». Comme la langue est le " premier instrument pour la civilisation d'une nation ", chaque camarade (shok) a " pour devoir d'aider les autres à éclairer leur esprit et à avoir un plus grand amour pour la patrie ". Il est aussi prévu que, lorsque le comité aura rassemblé assez d'argent, il enverra une ou deux personnes en Europe, afin d'expliquer ses buts aux Albanais ainsi qu'aux étrangers qui seraient prêts à aider. Comme les autres comités secrets de l'époque, il est organisé selon le principe des loges Carbonari. Par conséquent, chaque camarade doit trouver quatre nouveaux camarades qui, à leur tour, prêteront le serment de se vouer au comité, y compris en versant leur sang. L'égalité entre hommes et femmes

\footnotetext{
13 De fait, une commission internationale des finances avait été mise sur pied en 1905 pour régler le volet financier des réformes, après avoir été acceptée par la Porte à la suite d'une démonstration navale des Grandes Puissances.
}

14 vllamasi (Sejfi), op.cit., pp. 26-27.

15 HHStA, PA XIV/14, liasse XI, Prochaska, Monastir, 21/4/1906. 
est soulignée, bien que l'on ne possède aucune indication sur la participation de femmes ${ }^{16}$.

En réalité, les buts de cette nouvelle organisation appelée " Comité des Albanais pour la liberté de l'Albanie " ont pu être formulés de différentes manières, selon ses membres, leurs interlocuteurs et la période. Au printemps 1906, le consul austro-hongrois à Bitola rapporte que les revendications du comité sont : la reconnaissance officielle de la langue albanaise dans l'administration et à l'école, la mise en place de fonctionnaires albanais dans le pays et le non-envoi des troupes albanaises en dehors des provinces européennes des revendications qui sont donc davantage liées aux relations entre "Albanais " et gouvernement ottoman qu'à la promotion de l'union et du progrès parmi eux ${ }^{17}$.

Un an plus tard, Shahin Kolonja explique à un représentant de la Double Monarchie que le but du comité est de "ramener la population albanaise à la conscience de sa nationalité, dans les régions où elle est grécisée à travers l'activité du Patriarcat grec, c'est-à-dire au moyen de l'Église et des écoles, et d'engendrer un intérêt pour la langue albanaise $n^{18}$. Cette dernière affirmation, qui, au reste, rejoint les buts du comité tels qu'ils sont exprimés dans les statuts, appelle une remarque. L'objectif de cette organisation, dont les membres sont essentiellement des musulmans, serait donc, en particulier, de toucher les Albanais chrétiens orthodoxes et de s'opposer à la propagande grecque. La déclaration de Shahin Kolonja intervient, il est vrai, juste après l'assassinat du métropolite de Korçë par la çeta albanaise formée au printemps 1906.

De fait, à côté de la diffusion d'imprimés et de la propagande menée notamment parmi les soldats, les gendarmes et les policiers, le comité albanais a très tôt décidé de mettre sur pied des bandes, comme l'avaient fait les autres propagandes.

En mars 1906, afin d'organiser une première çeta, Bajo Topulli est entré en clandestinité avec trois étudiants de l'idadiye, accompagné de Hysejn bey Dishnica. Ils ont recruté dans la région de Kolonjë, au sud de Korçë, et dans la région de Gjirokastër, lieu de naissance de Bajo Topulli. Pendant plusieurs mois, les membres de ces deux groupes se sont contentés de distribuer de la littérature et de sensibiliser la population à la cause albanaise, tandis que d'autres sont partis convaincre les Albanais des diasporas de Bulgarie et de Roumanie de soutenir financièrement cette action. Mais, en septembre 1906, une bande dirigée par Çerçiz Topulli, le frère de Bajo, se signale par le meurtre

\footnotetext{
16 Komitet i Shqiptarëve për lirin' e Shqipërisë. Kanonizmë, s.I., s. d.

17 HHStA, PA XIV/14, liasse XI, Prochaska, Monastir, 21/4/1906.

${ }^{18}$ HHStA, PA XIV/13, Liasse VIII/1, Mitteilungen Schahin bey Kolonia über die Agitation Aladro's (1907).
} 
du métropolite de Korçë, donnant à l'action du comité une autre dimension.

Dans le numéro du 15 novembre du journal Drita, la photo de Bajo Topulli entouré de son frère et d'un autre membre de la bande apparait en première page. Elle accompagne un texte qui revendique le meurtre du prêtre, acte fondateur de la guérilla albanaise. Dans cet article, Bajo Topulli explique que cet assassinat n'est que l'expression de la vengeance, pour l'honneur et le bien de la nation, du meurtre de Papa Kristo de Negovan, assassiné par une bande grecque au début de l'année 1905. Lui-même a été offusqué du fait que ce meurtre n'ait pas été puni par le gouvernement ottoman. Plus tard, il a mème appris que deux Albanais qui ont donné de l'argent pour les orphelins de Papa Kristo ont été injustement arrètés ${ }^{19}$. C'est pour cette raison, explique-t-il, qu'il a fondé avec des patriotes une ligue pour venger le sang de ce pope albanais, serviteur de la nation. Il a ensuite quitté son poste à l'idadiye, s'est mis à distribuer des livres et a constitué une çeta de treize personnes, comprenant des musulmans et des chrétiens ${ }^{20}$. Puis, il a été chargé de "reprendre le sang " du pope $^{21}$.

Il est vrai que le journal Drita de Shahin Kolonja, que lisait probablement Bajo Topulli à Bitola, avait lancé une campagne à la suite du meurtre du pope, dénonçant la responsabilité de la Grèce et de l'Église grecque, taxées de barbares et de sauvages. Le journal avait contribué à élever Papa Kristo au rang de martyr (dëshmor) de la langue et de la nation, son rédacteur poussant les lecteurs à venger cet événement, comparable à celui de Kerbela, c'est-à-dire au martyre de Hüseyin, le petit-fils du Prophète, et dénonçant la barbarie grecque contre toute la nation albanaise et contre sa langue ${ }^{22}$. Au cours de l'été 1905, le périodique avait également lancé une campagne de collecte de fonds pour aider les enfants du martyr. En juillet 1906, une caricature représentant l'assassinat du pope figurait encore en première page de Drita. Parallèlement, le périodique avait entamé une campagne de diffamation contre le métropolite de Korçë.

Quel que soit l'impact véritable du meurtre de Papa Kristo, qui avait eu lieu un an et demi plus tôt, ou de celui de la feuille de Shahin Kolonja, en automne 1906, les membres du comité albanais ont donc voulu exprimer pu-

\footnotetext{
19 Notons qu'il s'agit donc aussi d'une réaction vis-à-vis de la justice et des autorités ottomanes.

20 En réalité, la çeta ne comprenait qu'un chrétien, Apostoll Plasa, du village de Plase, près de Korçë (Grameno (Mihal), Kryengritja shqiptare (La révolte albanaise), Vlorë, 1925, p. 28).

${ }^{21}$ Drita, (85), 15/11/1906, p. 1.

22 Voir Drita, (58), (59) et (60). Papa Kristo y ètait présenté comme la victime de l'Église et de la nation grecque, aidees par le gouvernement ottoman, ou encore comme la victime " des Grecs et des Grécomanes ", la victime " des barbares sauvages grecs et grécomanes ".
} 
bliquement une "solidarité nationale " par le biais d'une "vengeance ", non sans instrumentaliser la loi coutumière albanaise. Or, cette solidarité est d'autant plus manifeste qu'elle se fait au delà des frontières religieuses, ce que les orthodoxes ne sont d'ailleurs pas encore tout à fait prêts à admettre. Après ce coup d'éclat cependant, alors que les membres du comité à Bitola ont déjà été arrêtés depuis plusieurs mois, la çeta albanaise doit se dissoudre et ses chefs partent mobiliser les diasporas de Bulgarie, de Roumanie et des États-Unis ${ }^{23}$.

\section{LES ORTHODOXES ALBANAIS, LE PATRIARCAT ET LE « MEURTRE DU PRÊTRE »}

En 1905-1906, on l'a vu, les milieux orthodoxes albanophones sont encore très pétris d'hellénisme. L'opposition au clergé considéré comme " grec " et à une " orthodoxie grecque " ne peut revêtir des formes trop brutales. Dans le journal Kombi [La nation] essentiellement rédigé par des orthodoxes ${ }^{24}$, on voit que l'assassinat du métropolite de Korçë met les éditeurs dans l'embarras au contraire de ceux de Drita. Ils se demandent d'abord qui a pu être responsable d'un tel acte, tout en s'élevant contre les interprétations de la presse grecque qui accuse les Albanais musulmans, voulant ainsi, selon eux, diviser les Albanais. Les éditeurs de Kombi ne veulent pas voir dans cet assassinat une manifestation de leur " mouvement " à eux, les chrétiens. Ils affirment ne pas vouloir mener une lutte systématique contre les "Phanariotes " (ainsi appellent-ils les membres du clergé proches du Patriarcat). Ils finissent par expliquer que Photios n'a d'ailleurs pas été tué par les Albanais, mais par sa propre politique : il n'était pas un bon pasteur. Car ils critiquent aussi fortement l'attitude " anti-chrétienne " du " clergé grec fanatique ". Ils parlent mème de " joug grec $"$.

Peu à peu, ce sont pourtant eux, les éditeurs de Kombi, qui vont être des acteurs de premier plan, non pas dans une lutte armée, mais dans une nouvelle scission au sein de l'Église orthodoxe, en l'occurrence dans la naissance d'une Église orthodoxe albanaise aux États-Unis. Au printemps 1907, le périodique et la société albanaise Besa-besë dirigée par Fan Noli érigent la Saint-Georges

\footnotetext{
${ }^{23}$ Au printemps 1907 , une nouvelle bande est formée sous la direction de Çerçiz Topulli, dans laquelle un jeune albaniste chrétien de la diaspora, Mihal Grameno, s'enröle. Son activité consiste essentiellement à faire de la propagande dans les villages du vilayet de Ioannina et des sandjak macédoniens. Elle ne combat donc pas. Au printemps 1908, elle décide néanmoins de faire un nouveau coup d'éclat en assassinant le commandant de gendarmerie de Gjirokastër, ce qui l'entraine dans un court affrontement avec les troupes ottomanes (cf. Grameno (Mihal), op.cit.).
}

24 Ce périodique parait à Boston, aux États-Unis. Il est alors le second journal albanais, par l'importance de son lectorat. Ces principaux éditeurs sont Sotir Peci et Fan Noli. 
" qui était fêtée par les chrétiens et les musulmans " en " une célébration de la bravoure de Skanderbeg [initialement prénommé Gjergj, c'est-à-dire Georges], à l'époque où l'Albanie était louée dans toute l'Europe, lorsque les Albanais étaient unis sous un drapeau ". Il s'agit de célébrer religieusement et nationalement, comme les autres peuples, "le jour de la libération d'un joug extérieur ou intérieur ${ }^{25}$. Mais cette volonté de nationaliser le religieux va beaucoup plus loin, car, au même moment, les rédacteurs de Kombi suggèrent la constitution d'un comité, afin que les Albanais se dotent d'une église à Boston et d'un pope, afin de remplir un devoir " religieux et national ", en suivant l'exemple des autres colonies des États-Unis qui ont leurs journaux et églises, à l'instar des Valaques, c'est-à-dire des Aroumains.

Cette idée met un an à cheminer et, pour diverses raisons, finit par conduire à la formation d'un embryon d'Église albanaise indépendante. Jugeant qu'il faut utiliser la liberté religieuse qui existe aux États-Unis pour réaliser ce que les Albanais de Roumanie veulent depuis longtemps faire, c'està-dire " poser les fondements d'une Église albanaise pour l'intérieur de l'Albanie, pour la libération du joug de l'Église grecque ", un petit groupe d'albanistes envoie alors Fan Noli auprès de Monseigneur Platon, évêque russe de New York. En février 1908, ce dernier accepte d'ordonner Fan Noli qui est consacré le 8 mars et célèbre, le 22 du même mois, la première messe en albanais dans l'église de Saint-Georges à Boston. Fan Noli se promet de traduire les textes liturgiques en albanais et d'être l'initiateur d'une "Église indépendante des instruments de la Grèce, des Phanariotes ${ }^{26}$.

Or, on constate que c'est précisément lorsque les éditeurs de Kombi décident, vers la fin de l'année 1907, de couper le cordon ombilical qui les relie au Patriarcat, qu'ils modifient leur façon de voir l'assassinat du métropolite. Désormais, ils justifient le meurtre : l'assassinat de Photios a été exécuté selon la coutume du sang, afin de venger le meurtre de Papa Kristo Negovani. Mais, ils tiennent à préciser qu'il ne s'agit pas d'un acte dirigé contre le "clergé grec " en tant que tel. Pourtant, ils laissent paraître une réaction à un article paru dans la revue Albania de Faik Konica ${ }^{27}$ rédigée par un Albanais d'Égypte, dans laquelle ce dernier explique que les " prêtres grecs " avaient tué le seul "prêtre albanais ". Bien que musulman, c'est Bajo Topulli qui avait vengé, de façon juste et nécessaire, ce meurtre d'un innocent ayant seulement déclaré qu'il était albanais. Et d'ajouter que les métropolites n'étaient pas des personnes désarmées, puisqu'ils collaboraient avec les vali et les pachas.

\footnotetext{
$25 \mathrm{Kombi}$, (34) et (35) (26/04 et 03/05/1907). Cet essai de faire de la Saint-Georges une fête nationale n'aura pas de suite dans le cadre de l'Albanie indépendante.

${ }^{26} \mathrm{Kombi},(25),(51),(52),(53),(56),(60),(62),(67),(72)$.

27 Albania (Londres), 10 (8), 1906. Dans l'article, Bajo Topulli était sévèrement critiqué pour avoir tué un homme sans défense.
} 
Le commentaire des éditeurs de Kombi nous montre comment, au fil des mois et des événements, ils ont, eux aussi, adopté une attitude plus radicale. Ils expliquent en effet qu'ils ont toujours dit qu'il ne fallait pas utiliser la violence contre la propagande grecque et que, dans ce sens, la revue Albania a raison. Cependant, pour eux, la situation est telle qu'une propagande pacifiste ne convient plus : le gouvernement a exilé tous les " patriotes " et la propagande grecque ne se contente plus d'utiliser les écoles et les églises ; elle emploie dorénavant aussi la force. En conséquence, Bajo Topulli a été " l'épée salvatrice " nécessaire contre "l'épée grecque ${ }^{28}$.

\section{CONCLUSION}

Le meurtre du prêtre, ou plus exactement du plus haut représentant de la hiérarchie ecclésiastique orthodoxe de la région de Korçë, revêt assurément un caractère inaugural. Avec lui, la mobilisation albanaise clandestine qui vient de se constituer quelques mois auparavant se manifeste sur la scène politique. Le message s'adresse aux autorités ottomanes, qui sont accusées de ne pas garantir la justice. Il s'adresse aussi au Patriarcat de Constantinople et à la hiérarchie orthodoxe, sans oublier les autorités de l'État grec accusées de collaborer étroitement avec cette hiérarchie. Mais, ce témoignage d'un engagement pouvant faire acte de violence est également un message adressé à la population de la région dans son ensemble. À ceux qui voudraient collaborer avec la hiérarchie patriarchiste "pro-grecque ", il signifie que, puisque cette autorité a été touchée, tous ceux qui suivent sa ligne peuvent l'être également. Aux autres, il indique que, désormais, la solidarité entre musulmans et chrétiens, au sein d'une nation albanaise, est chose concrète et donc que la frontière religieuse ne peut, en aucune manière, empêcher l'existence de cette nation.

Dans le choix de la cible, on peut également déceler, en toile de fond, une opposition entre porteurs de la mobilisation nationaliste et Églises, comme Bernard Lory le souligne dans sa contribution. J'apporterai une nuance, car il semble surtout que les premiers souhaitent voir les secondes dans une relation de subordination. Le rejet, puis l'instrumentalisation du meurtre par des albanistes orthodoxes au moment où il s'agissait de dissocier orthodoxie et hellénisme, en forgeant une expression albanaise de l'orthodoxie et en allant vers la création d'une Église orthodoxe albanaise, le montre.

${ }^{28}$ Voir les numéros 17 (de 1906), 21 et 22 (de janvier 1907), 64 (de décembre 1907), 70 et 77 (de 1908) du journal Kombi. 